\title{
Sum rules and correlations in asymmetric nuclear matter
}

\author{
A. Rios and A. Polls \\ Departament d'Estructura i Constituents de la Matèria, Universitat de Barcelona, E-08028 Barcelona, Spain \\ H. Müther \\ Institut für Theoretische Physik, Universität Tübingen, D-72076 Tübingen, Germany
}

(Received 25 July 2005; published 8 February 2006)

\begin{abstract}
The neutron and proton single-particle spectral functions in asymmetric nuclear matter fulfill energy-weighted sum rules. The validity of these sum rules within the self-consistent Green's function approach is investigated. The various contributions to these sum rules and their convergence as a function of energy provide information about correlations induced by the realistic interaction between the nucleons. The study of the sum rules in asymmetric nuclear matter exhibits the isospin dependence of the nucleon-nucleon correlations.
\end{abstract}

DOI: 10.1103/PhysRevC.73.024305

PACS number(s): 21.65.+f, 21.30.Fe

\section{INTRODUCTION}

The microscopic description of the single-particle properties in nuclear matter must deal with the treatment of nucleon-nucleon $(N N)$ correlations [1,2]. In fact, the strong short-range and tensor components that are required in realistic $N N$ interactions to fit the $N N$ scattering data lead to corresponding correlations in the nuclear wave function. These $N N$ correlations are important to describe bulk properties of dense matter. They also modify the spectral distribution of the singleparticle strength in a significant way. Recent calculations have demonstrated without ambiguity that $N N$ correlations produce a partial occupation of the single-particle states, which are completely occupied in the mean-field approach, and a wide distribution of the single-particle strength in energy. These two features have found experimental grounds in the analysis of $\left(e, e^{\prime} p\right)$ reactions $[3,4]$.

Historically, various tools have been employed to account for correlations in the nuclear many-body wave function. These include the traditional Brueckner hole-line expansion [5] and variational approaches using correlated basis functions [6]. Attempts have also been made to employ the technique of a self-consistent evaluation of Green's functions (SCGF) $[1,2,7,8]$ to the solution of the nuclear many-body problem. This method offers various advantages: (i) the single-particle Green's function contains detailed information about the spectral function, i.e., the distribution of single-particle strength as a function of missing energy and momentum; (ii) the method can be extended to finite temperatures, a feature that is of interest for the study of the nuclear properties in astrophysical environments; and (iii) the Brueckner-Hartree-Fock (BHF) approximation, the approximation to the hole-line expansion that is commonly used, can be considered as a specific prescription within this scheme.

Enormous progress in the SCGF applications to nuclear matter has been achieved in the past several years, at both zero [9] and finite temperatures [10-13]. The efforts at $T=0$ are mainly oriented to provide the appropriate theoretical support for the interpretation of the $\left(e, e^{\prime} p\right)$ experiments, whereas research at finite $T$ is essentially focused on the description of the nuclear medium in astrophysical environments or on the interpretation of heavy-ion collisions dynamics. In all cases, a key quantity is the single-particle spectral function that measures the possibility of adding or removing a particle with a given momentum at a specific energy. A useful way to study the properties of these single-particle spectral functions is by means of the energy-weighted sum rules, which are well established in the literature and which have been numerically analyzed for symmetric nuclear matter at zero [14] and finite temperatures [15].

Recently, the single-particle spectral functions in hot asymmetric nuclear matter have been calculated within the SCGF framework [13]. In this computation, the bulk properties of asymmetric dense matter were computed, namely the energy per particle, the symmetry energy, and the chemical potentials. In addition, and because the SCGF approach gives access to the correlated momentum distributions of neutrons and protons, the influence of the asymmetry on the depletion of the momentum states could be studied. In neutron-rich matter the proton states with momenta below the Fermi momentum are more strongly depleted than in the symmetric system at the same density. In this case the occupation of neutron states within their Fermi sphere are enhanced compared to the symmetric case. This indicates that the proton-neutron interaction is a stronger source of correlation as compared to proton-proton and neutron-neutron interaction.

In this article, we want to use the energy weighted sum rules to investigate the dependence of the single-particle spectral functions on the asymmetry more in detail. These sum rules may help to explore the isospin dependence of the short-range correlations in asymmetric matter. Note that the proton-proton and neutron-neutron correlations are predominantly determined by the central short-range repulsion in the ${ }^{1} S_{0}$ partial wave, whereas the proton-neutron correlations receive a sizable contribution from the tensor interaction in the ${ }^{3} S_{1}-{ }^{3} D_{1}$ partial wave. Moreover, the analysis of the energy-weighted sum rules provides valuable tests on the numerical accuracy of our calculations. All the computations discussed in this article are performed in the framework of SCGF employing a fully self-consistent ladder approximation in which the complete spectral function has been used to describe the intermediate states in the Galistkii-Feynman equation $[12,13]$. 
After this introduction, we recall the energy-weighted sum rules in Sec. II. The results obtained for hot asymmetric nuclear matter using the charge-dependent Bonn potential CDBONN [16] are presented in Sec. III, where a short summary of the main conclusions is given as well.

\section{SUM RULES FOR ASYMMETRIC NUCLEAR MATTER}

In the $T$-matrix approximation to the self-energy reported in Ref. [13], one can determine the single-particle Green's function as the solution of Dyson's equation for any complex value of the frequency variable $z$ as follows:

$$
g_{v}(k, z)=\frac{1}{z-\frac{k^{2}}{2 m}-\Sigma_{v}(k, z)} .
$$

The analytical properties of the finite temperature Green's function allows one to derive the corresponding Lehmann representation, which for slightly complex values of the frequency can be written as follows:

$$
g_{\nu}(k, \omega+i \eta)=\int_{-\infty}^{+\infty} \frac{d \omega^{\prime}}{2 \pi} \frac{A_{\nu}\left(k, \omega^{\prime}\right)}{\omega-\omega^{\prime}+i \eta} .
$$

Here and in the following $A_{v}(k, \omega)$ denotes the spectral function for protons or neutrons (label $v$ ) with momentum $k$ in asymmetric nuclear matter at a finite temperature. The spectral function $A_{v}(k, \omega)$ is split into the sum of the two positive functions, $A_{v}^{<}$and $A_{v}^{>}$,

$$
A_{v}(k, \omega)=A_{v}^{<}(k, \omega)+A_{v}^{>}(k, \omega),
$$

using the relation

$$
\begin{aligned}
& A_{v}^{<}(k, \omega)=f_{v}(\omega) A_{v}(k, \omega), \\
& A_{v}^{>}(k, \omega)=\left[1-f_{v}(\omega)\right] A_{v}(k, \omega),
\end{aligned}
$$

where $f_{v}(\omega)$ is the Fermi-Dirac distribution function for the temperature under consideration. The integration of the function $A_{v}^{<}$leads to the occupation probability

$$
n_{v}(k)=\int_{-\infty}^{+\infty} \frac{d \omega}{2 \pi} A_{v}^{<}(k, \omega),
$$

for the state with isospin $v$ and momentum $k$.

The functions $A_{v}^{<}$and $A_{v}^{>}$can be compared with the hole and particle part of the spectral function at zero temperature. In particular, the limit of $A_{v}^{<}$at zero temperature reads as follows:

$$
A_{v}^{h}=2 \pi \sum_{r}\left|\left\langle\Psi_{r}^{A-1}\left|a_{k v}\right| \Psi_{0}^{A}\right\rangle\right|^{2} \delta\left[\omega-\left(E_{0}^{A}-E_{r}^{A-1}\right)\right],
$$

where $\left|\Psi_{0}^{A}\right\rangle$ is the ground-state with $N_{n}$ neutrons and $N_{p}$ protons (such that $A=N_{p}+N_{n}$ ) and $\left|\Psi_{r}^{A-1}\right\rangle$ labels the excited energy state of a system with a neutron or a proton less (depending on which type of annihilation operator $a_{k v}$ has been applied to the ground state). By its own definition, it is clear that the lowest possible energy of the final state is the ground-state energy of the $A-1$ particle system, so that there is an upper limit for the hole spectral function $\omega_{\nu}=E_{0}^{A}-E_{0}^{A-1_{v}}=\mu_{\nu}$, both for neutrons and protons. In a similar way one can define the particle part of the spectral function and find a lower bound for the excitation energy of the $A+1$ particle system, measured with respect to the ground state of the $A$ particle system. At zero temperature, the existence of these lower and upper bounds causes a complete separation in energy between the particle and the hole part of the spectral function.

The sum rules for the spectral functions can be derived from the asymptotic behavior at large $\omega$ by expanding the real part of both previous expressions for the Green's function, Eqs. (1) and (2), in powers of $1 / \omega$. This yields the following:

$\operatorname{Re} g_{v}(k, \omega)=\frac{1}{\omega}\left\{1+\frac{1}{\omega}\left[\frac{k^{2}}{2 m}+\lim _{\omega \rightarrow \infty} \operatorname{Re} \Sigma_{v}(\mathrm{k}, \omega)\right]+\cdots\right\}$

and

$$
\begin{aligned}
\operatorname{Re} g_{v}(k, \omega)= & \frac{1}{\omega}\left\{\int_{-\infty}^{+\infty} d \omega^{\prime} A_{v}\left(k, \omega^{\prime}\right)\right. \\
& \left.+\frac{1}{\omega} \int_{-\infty}^{+\infty} d \omega^{\prime} \omega^{\prime} A_{v}\left(k, \omega^{\prime}\right)+\cdots\right\} .
\end{aligned}
$$

By comparing the first two expansion coefficients, one finds the $m_{0}$

$$
\int_{-\infty}^{+\infty} \frac{d \omega}{2 \pi} A_{v}(k, \omega)=1,
$$

and the $m_{1}$ sum rules

$$
\int_{-\infty}^{+\infty} \frac{d \omega}{2 \pi} \omega A_{v}(k, \omega)=\frac{k^{2}}{2 m}+\lim _{\omega \rightarrow \infty} \operatorname{Re} \Sigma_{v}(k, \omega) .
$$

Is is worth mentioning that in our scheme the self-energy is derived in the $T$ matrix approximation and so its real part is computed from the imaginary part using the following dispersion relation:

$\operatorname{Re} \Sigma_{v}(k, \omega)=\Sigma_{v}^{\infty}(k)-\frac{\mathcal{P}}{\pi} \int_{-\infty}^{+\infty} d \lambda \frac{\operatorname{Im} \Sigma_{v}(k, \lambda+i \eta)}{\omega-\lambda}$.

In the derivation of the previous equation, the spectral decomposition of the Green's function is already used, so it is a property of the $T$-matrix approach that it fulfills the sum rule. Nevertheless, the sum rules still provide a useful consistency check for the numerics. The first term on the right-hand side of Eq. (11) is the energy-independent part of the self-energy,

$$
\Sigma_{\nu}^{\infty}(k)=\sum_{\tau} \int \frac{d^{3} k^{\prime}}{(2 \pi)^{3}}\left\langle\mathbf{k} v \mathbf{k}^{\prime} \tau|V| \mathbf{k} v \mathbf{k}^{\prime} \tau\right\rangle_{A} n_{\tau}\left(\boldsymbol{k}^{\prime}\right)
$$

which can be identified with the $\operatorname{limit}_{\lim _{\omega \rightarrow \infty}} \operatorname{Re} \Sigma_{v}(k, \omega)$, because the dispersive part decays like $1 / \omega$ for $\omega \rightarrow \pm \infty$. Equation (12) looks like a Hartree-Fock potential. However, $n_{v}(k)$ is the momentum distribution of Eq. (5) containing the depletion effects because of $N N$ correlations and temperature.

\section{RESULTS AND DISCUSSION}

All the results discussed in this article have been obtained with the charge-dependent Bonn (CDBONN) potential. Because we want to focus our study on the dependence of the observables on asymmetry, we consider only one density $\rho=0.16 \mathrm{fm}^{-3}$ and one single temperature $T=5 \mathrm{MeV}$. This temperature is low enough to allow for conclusions in the limit of zero temperature but high enough to avoid instabilities 
associated with neutron-proton pairing [17-19]. As for the proton fractions, we consider three different cases. The first one corresponds to symmetric nuclear matter $x_{p}=\rho_{p} / \rho=0.50$ and serves as a guideline for the other cases. The last one has a very low proton fraction $x_{p}=0.04$ and corresponds to the $\beta$-stable composition of matter with nucleons, electrons, and muons at $T=5 \mathrm{MeV}$ and $\rho=0.16 \mathrm{fm}^{-3}$. This composition can be computed thanks to the fact that we know the asymmetry dependence of both the neutron and the proton chemical potentials at this density [13]. Finally, we consider an intermediate fraction $x_{p}=0.30$, which is useful in identifying the effects for low asymmetries of the system.

We start by discussing the momentum dependence of the single-particle spectral functions of neutrons and protons. Depending on how far above or below the considered momentum is from the Fermi momentum of the corresponding particle, we expect a very different behavior for the spectral function. Figure 1 shows the neutron (left panels) and proton (right panels) single-particle spectral functions for three different momenta $\left(k=0, k_{F}^{v}\right.$ and $2 k_{F}^{v}$, with $k_{F}^{v}$ the Fermi momentum of each nucleon species) at a proton fraction of $x_{p}=0.04$. The dotted vertical line corresponds to the point where $\tilde{\omega}=-\mu_{\nu}$. Since the variable $\tilde{\omega}$ is defined as $\tilde{\omega} \equiv \omega-\mu_{\nu}$, the line shows the point where $\omega=0$, and thus it indicates the point below which the contribution to the sum rule $m_{1}$ in Eq. (10) is negative.

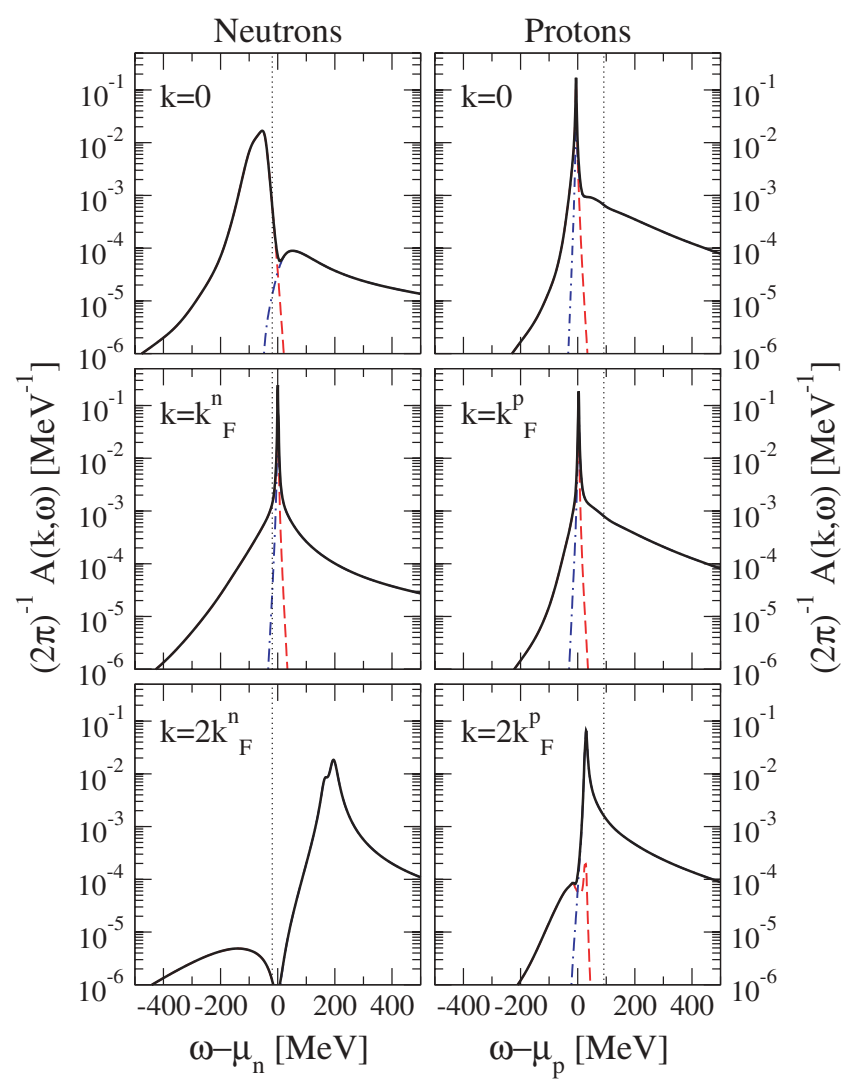

FIG. 1. (Color online) Neutron (left panels) and proton (right panels) single-particle spectral functions at $\rho=0.16 \mathrm{fm}^{-3}, T=$ $5 \mathrm{MeV}$ and proton fraction $x_{p}=0.04$ for three different momenta. Both $A_{v}^{<}$(dashed lines) and $A_{v}^{>}$(dot-dashed lines) are displayed together with the total spectral function $A_{v}$ (solid lines).
The contributions $A_{v}^{<}$and $A_{v}^{>}$are given by the dashed and dash-dotted lines, respectively. Because we are considering finite temperatures, thermally excited states are always included in the definition of the spectral function. This leads to contributions to $A_{v}^{<}$at energies $\omega$ larger than $\mu_{v}$ ( $\tilde{\omega}$ larger than zero). Similarly, $A_{v}^{>}(k, \omega)$ extends to the region below $\mu_{v}$. In general there is no longer a clear separation in energy between $A_{v}^{>}$and $A_{v}^{<}$, as it is the case for $T=0$. Actually, the maxima of $A_{v}^{>}$and $A_{v}^{<}$can even coincide.

For the case of neutrons at $k=0$, the peak of the spectral function is provided by $A_{n}^{<}$. This is due to the fact that the position of this peak, which can be identified with the quasiparticle energy $\epsilon_{q p}^{n}(k)$, is well below the chemical potential $\mu_{n}$, which implies that the value of Fermi-Dirac distribution function $f(\omega)$ at this energy $\omega=\epsilon_{q p}^{n}(0)$ is very close to one [see Eq. (5)]. Because the quasiparticle peak is well below $\mu_{n}$, the thermal effects do not fill up the minimum in the spectral function at $\omega=\mu_{n}$ and we still observe some kind of separation in energy between $A_{n}^{<}$and $A_{n}^{>}$. However, around $\tilde{\omega}=0$ there is an energy interval, in which both $A_{n}^{<}$ and $A_{n}^{>}$are small but different from zero, i.e., an interval in which these functions overlap.

A similar situation is observed for the neutron spectral function at $k=2 k_{F}^{n}$. In this case the quasiparticle energy is well above $\mu_{n}$, which means that $[1-f(\omega)]$ is very close to one at this energy and the peak structure is supplied by $A_{n}^{>}$ [see Eq. (5)]. Therefore we observe a clear separation between the hole part $\left(A_{n}^{>}\right)$and the particle part $\left(A_{n}^{<}\right)$of the spectral function even at finite temperature.

At $k=k_{F}^{n}$, the quasiparticle peak is close to the chemical potential. This means that $f\left[\epsilon_{q p}^{n}(k)\right]$ is around 0.5 and therefore $A_{n}^{<}$and $A_{n}^{>}$have a peak at $\tilde{\omega}=0$. The thermal effects fill up the zero that the spectral function has at $\tilde{\omega}=0$ in the $T=0$ limit and the overlap region of $A_{n}^{<}$and $A_{n}^{>}$is enhanced.

In the case of a very small proton fraction, like $x_{p}=0.04$, the Fermi momentum $k_{F}^{p}$ is rather small and consequently the quasiparticle energies are close to the Fermi energy $\mu_{p}$ for $k=k_{F}^{p}$ as well as $k=0$ and $k=2 k_{F}^{p}$. Therefore, although the relative distances to the Fermi surfaces are the same, there are strong overlaps between $A_{p}^{<}$and $A_{p}^{>}$at all three momenta. Even in the case of $k=2 k_{F}^{p}$, a small peak structure for $A_{p}^{<}$ around $\tilde{\omega}=0$ can be observed.

In Table I (neutrons) and Table II (protons) we report the fraction of the integrated strength of $A_{v}^{<}$below and above the corresponding chemical potential, $\mu_{\nu}$. In fact, if we identify $A_{v}^{<}$with the $T=0$ hole spectral function, the integrated strength above the chemical potential would be exactly zero (because $A_{v}^{<}=0$ for $\omega>\mu_{v}$ ). In this sense, the integrated strength above the chemical potential can be considered as a genuine thermal effect. As expected, these thermal effects are important around $k_{F}^{v}$, where the overlap between $A_{v}^{<}$and $A_{v}^{>}$is significant. Notice also that for this very neutron-rich system, neutrons are less affected by temperature, whereas protons (which have a substantially lower Fermi momentum and can thus be considered as a dilute system) are much more influenced by temperature. For instance, protons show a large amount of strength above $\mu_{F}^{p}$ up to momenta $k / k_{F}^{p} \sim 2$. For the sake of completeness, the corresponding occupation numbers for each species are also listed in those tables. 
TABLE I. Strength distribution of $A_{n}^{<}$at $\rho=0.16 \mathrm{fm}^{-3}, T=$ $5 \mathrm{MeV}$ and proton fraction $x_{p}=0.04$. The numbers give the fraction of the integrated strength above and below the neutron chemical potential $\mu_{n}$. The last column reports the occupation of the respective neutron momentum state.

\begin{tabular}{lccc}
\hline \hline$k / k_{F}^{n}$ & Below $\mu_{n}[\%]$ & Above $\mu_{n}[\%]$ & $n(k)$ \\
\hline 0.0 & 99.98 & 0.02 & 0.971 \\
0.5 & 99.96 & 0.04 & 0.960 \\
1.0 & 55.58 & 44.42 & 0.464 \\
1.5 & 98.48 & 1.52 & 0.006 \\
2.0 & 99.76 & 0.24 & 0.001 \\
\hline \hline
\end{tabular}

The $m_{0}$ sum rule is fulfilled with an accuracy better than $0.1 \%$ for both neutrons and protons in the complete momentum range. Results for $m_{1}$ both for neutrons (upper panels) and protons (lower panels) are reported in Fig. 2 at the three proton fractions $x_{p}=0.5,0.3$, and 0.04 . The vertical dotted lines indicate the location of $k_{F}^{v}$ for each case. Because the sum rule is satisfied better than $1 \%$ in all cases, the left- and the right-hand sides of Eq. (10) (solid lines) lie on top of each other and cannot be distinguished. To understand the $k$ dependence of the different contributions to $m_{1}$, it is useful to keep in mind the proton and neutron chemical potentials at the different concentrations given in Table III.

The lower dash-dotted line shows the $m_{1}$ contribution from $A_{v}^{<}$. For momenta below $k_{F}^{v}$, the contribution of $A_{v}^{<}$to $m_{1}$ is dominated by the quasiparticle peak, which lies below $\mu_{\nu}$. As the momentum increases, the peak appears closer to $\omega=0$ and its weight in the integral is diminished, which makes the integral smaller in magnitude and thus the contribution to the sum rule becomes an increasing function of $k$. When we get closer to the Fermi momentum $k_{F}^{v}$, the peak moves to $\omega \sim \mu_{\nu}$ and the position of the chemical potential becomes crucial. As far as the chemical potential is negative, the contribution of $A_{v}^{<}$will be negative because the Fermi-Dirac function falls off
TABLE II. Strength distribution of $A_{p}^{<}$at $\rho=0.16 \mathrm{fm}^{-3} T=$ $5 \mathrm{MeV}$ and proton fraction $x_{p}=0.04$. The numbers give the fraction of the integrated strength above and below the proton chemical potential $\mu_{p}$. The last column reports the occupation of the respective proton momentum state.

\begin{tabular}{lccc}
\hline \hline$k / k_{F}^{p}$ & Below $\mu_{p}[\%]$ & Above $\mu_{p}[\%]$ & $n(k)$ \\
\hline 0.0 & 97.62 & 2.38 & 0.605 \\
0.5 & 94.88 & 5.12 & 0.531 \\
1.0 & 21.62 & 78.38 & 0.280 \\
1.5 & 24.26 & 75.74 & 0.056 \\
2.0 & 64.10 & 35.90 & 0.009 \\
\hline \hline
\end{tabular}

close to $\mu_{v}$ and the integral will only catch a little positive zone if the chemical potential is close to $\omega \sim 0$. If, however, the chemical potential is positive, the integrand is not zero for $\omega>0$ and there can be a nonnegligible positive contribution to the integral when the quasiparticle peak lies between $\omega=0$ and $\omega=\mu_{\nu}$. This cancellations between positive and negative contributions give rise to the structures observed for the $A_{v}^{<}$ sum rule close to the Fermi momentum. In the upper right panel of Fig. 2, for instance, the asymmetry is so extreme that the neutron chemical potential $\mu_{n}>0$ and the positive contribution to the integral is important enough to pull the sum rule to positive values for $k \sim k_{F}^{n}$. Finally, for high momenta, $A_{v}^{<}$is strongly suppressed (the quasiparticle peak lies in the region $\omega>\mu_{v}$, which is suppressed by the Fermi-Dirac factor) and the contribution to the sum rule goes to zero.

The upper dash-dotted line displays the contribution from $A_{v}^{>}$. Because of the short-range correlations, there is always a high-energy tail that gives rise to a positive contribution that, for momenta well below $k_{F}^{v}$, is nearly constant. In the case of the neutron sum rules, this constant decreases slightly with decreasing proton fraction, although the chemical potential $\mu_{n}$ increases significantly. Such a small decrease is in accordance with the fact that the neutron-proton correlations are dominant

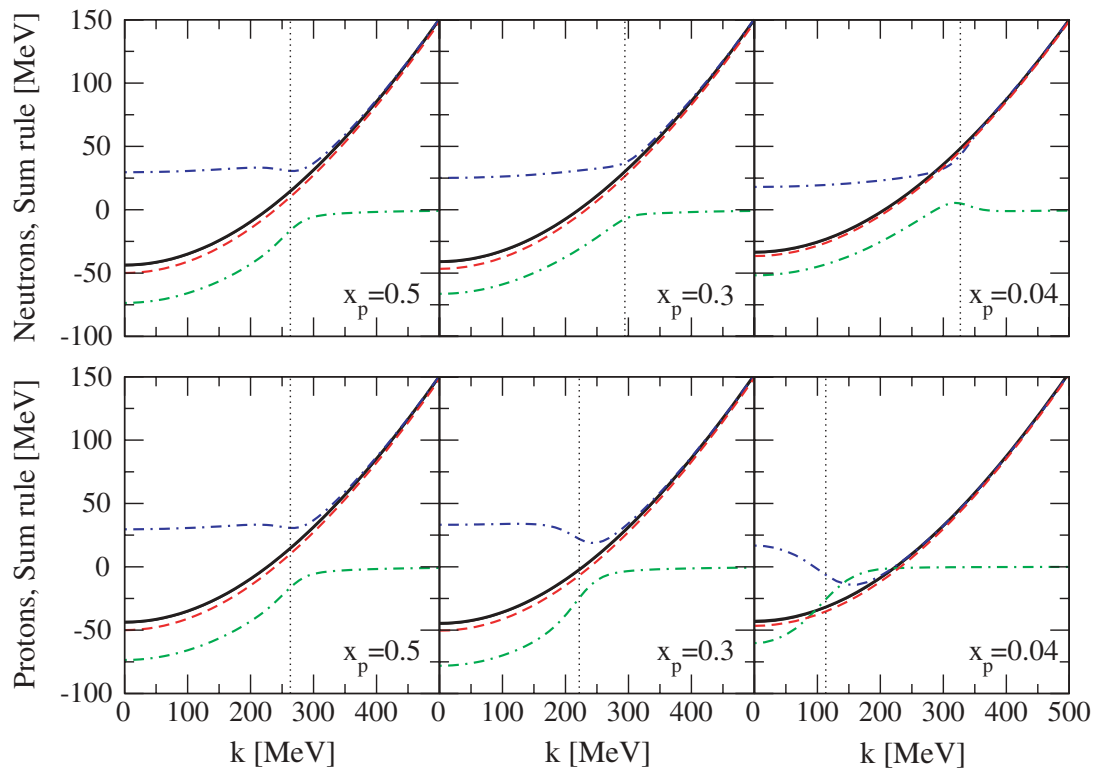

FIG. 2. (Color online) Energy-weighted sum rule $m_{1}$ (solid lines) for neutrons (panels above) and protons (panels below) at $\rho=$ $0.16 \mathrm{fm}^{-3}, T=5 \mathrm{MeV}$ and several proton fractions. Both the right- and the left-hand sides of Eq. (10) are displayed, but the sum rule is so well fulfilled that they cannot be distinguished. The contributions to $m_{1}$ that come from $A_{v}^{>}$and $A_{v}^{<}$are indicated by the upper and the lower dash-dotted lines, respectively. The result for the energy-weighted sum rule obtained from the Hartree-Fock approximation is represented by the dashed lines. 
TABLE III. Neutron and proton chemical potentials at $\rho=$ $0.16 \mathrm{fm}^{-3}, T=5 \mathrm{MeV}$ and different proton fractions.

\begin{tabular}{lcc}
\hline \hline$x_{p}$ & $\mu_{n}[\mathrm{MeV}]$ & $\mu_{p}[\mathrm{MeV}]$ \\
\hline 0.50 & -23.46 & -23.46 \\
0.30 & -2.78 & -48.33 \\
0.04 & 19.21 & -91.45 \\
\hline \hline
\end{tabular}

compared to the neutron-neutron ones. These high-energy tails (which are mainly caused by short-range correlations) are, nevertheless, not much affected by asymmetry. When the momentum becomes larger than $k_{F}^{n}, m_{1}$ increases monotonously following the location of the quasiparticle peak, which moves to higher energies when the momentum grows.

Also for protons one observes a contribution from $A_{v}^{>}$to the sum rule that is almost a constant for momenta smaller than the Fermi momentum. In this case, however, this constant is almost independent of the proton fraction, although the chemical potential $\mu_{p}$ gets significantly more attractive with decreasing $x_{p}$. At small values of $x_{p}$, the neutron abundance is large and this leads to strong correlation effects in the proton spectral function. In particular at $x_{p}=0.04$, which is the case corresponding to the lowest Fermi momenta, the total contribution of $A_{p}^{>}$is the result of a balance between positive and negative contributions. These can result in a minimum for $k \sim k_{F}^{p}$. In that particular proton fraction (see Fig. 1), the quasiparticle peaks always lie below $\omega=0$ and thus it give rise to a negative contribution. The relative width of this peak and the detailed structure of the high-energy tail can push the integral to negative values (almost $-15 \mathrm{MeV}$ at $k \sim$ $160 \mathrm{MeV}$ ). At larger momenta, however, the contribution to $m_{1}$ of $A_{p}^{>}$starts to grow steadily. Notice that for this asymmetry we cannot say that this is caused by the movement of the quasiparticle peak, because even at $k=2 k_{F}^{p}$ the quasiparticle peak lies in the region where the contribution to the integral is negative. The growth in $k$ is, to a large extent, a consequence of the contributions to the spectral functions at large energies.

In addition to these contributions, we have plotted in the dotted lines of Fig. 2 the Hartree-Fock approximation of $m_{1}$ at the same temperature, density, and proton fraction. This approximation turns out to give a very good estimate of $m_{1}$, an interesting result which has been already observed in the previous analysis for symmetric nuclear matter $[14,15]$. This result allows for a quantitative estimate of the amount of correlations produced by a given $N N$ potential without performing sophisticated many-body calculations.

Furthermore, it is worth noticing that the value of $m_{1}$ (specially for large $k$ ) is not so much affected by the asymmetry of the system. It is very similar for both neutrons and protons in all the three cases considered. This is a somewhat surprising result, because we have seen that the separate contributions of $A_{v}^{<}$and $A_{v}^{>}$can indeed be very different. Of course, at high momentum the kinetic energy is dominant and this could explain this fact in part. However, even at zero momentum the effect of the asymmetry is rather moderate, as we can see from Fig. 3, where $\Sigma_{v}^{\infty}, \Sigma_{v}^{\mathrm{HF}}$ (the Hartree-Fock approximation to the self-

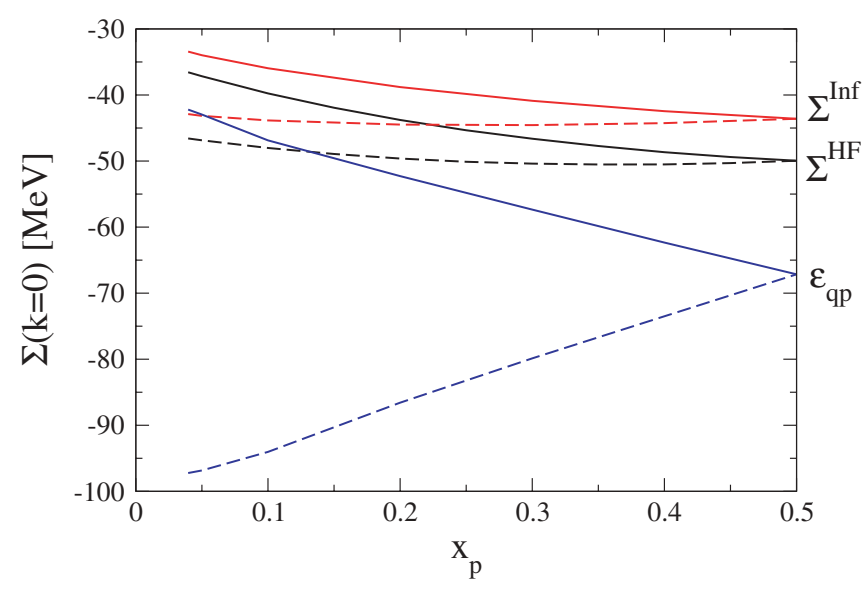

FIG. 3. (Color online) $\Sigma_{v}^{\infty}, \Sigma_{v}^{\mathrm{HF}}$ and $\epsilon_{q p}^{v}$ at zero momentum as a function of the proton fraction at $\rho=0.16 \mathrm{fm}^{-3}$ and $T=5 \mathrm{MeV}$ for neutrons (solid lines) and protons (dashed lines).

energy) and the quasiparticle energy $\epsilon_{q p}^{v}$ at zero momentum are plotted as a function of the proton fraction. Let us consider the highly asymmetric case of $x_{p}=0.04$. The isospin splitting of the Hartree-Fock energies $(k=0)$ at this asymmetry is around $10 \mathrm{MeV}$, which is rather small compared to the isospin splitting of the quasiparticle energies, which is around $55 \mathrm{MeV}$. This means that the stronger binding of the proton states as compared to the neutron states is to a small extent due to the attractive neutron-proton interaction in the bare $N N$ potential, the Born term of the $T$ matrix. The obtained attraction is then mainly caused by the terms in the $T$ matrix coming from the second and higher orders in the $N N$ potential. In other words, it is an effect of strong correlations in the isospin equal to zero $N N$ channels (like the ${ }^{3} S_{1}-{ }^{3} D_{1}$ partial wave), which lead to the deeply bound quasiparticle energy for protons at this large neutron abundance. These correlation effects, however, are also important for a redistribution of single-particle strength for protons with $k=0$ to energies well above the Fermi energy, leading to a low occupation probability (see Table II) and a large positive contribution to the sum rule $m_{1}$. This positive contribution yields to a value of $\Sigma^{\infty}$, which is even above the HF energy. The effects of correlations for the corresponding energies of the neutron state are much weaker, indicating that correlation effects in the isospin one $N N$ channels are weaker.

So we observe a compensation of correlation effects in the energy weighted sum rule. On the one hand, correlations lead to a more attractive quasiparticle energy (compared to the HF result) and therefore to a more attractive contribution of the quasiparticle pole to the sum rule. On the other hand, correlations shift single-particle strength to high energies, which yield a repulsive contribution to $m_{1}$. This compensation of correlation effects explains that the value of the energy integrated sum rule for the correlated system can be very similar to the HF result.

This compensation of correlation effects can also be observed in Fig. 4, which shows the exhaustion of the sum rules $m_{0}$ (left panel) and $m_{1}$ (right panel) at a momentum $k=$ $400 \mathrm{MeV}$ for both neutrons (solid lines) and protons (dashed 

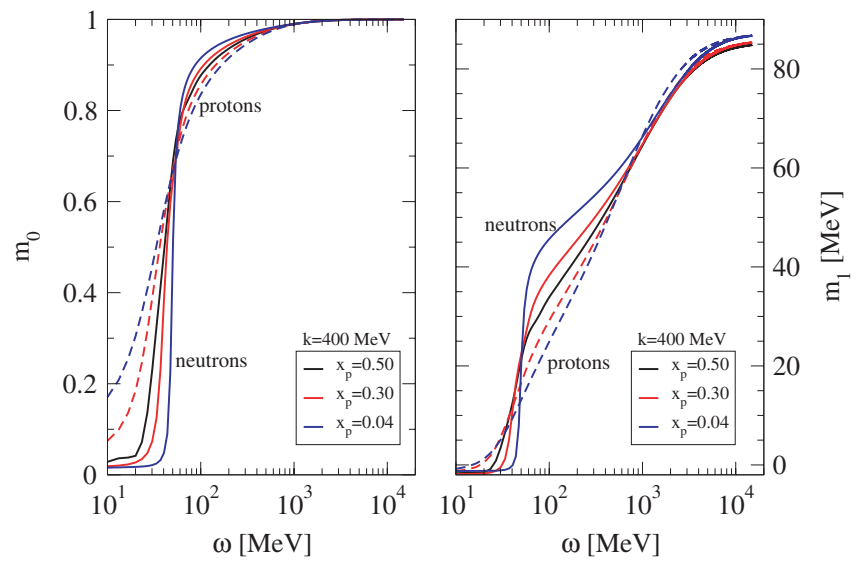

FIG. 4. (Color online) Saturation of the sum rules $m_{0}$ (right panel) and $m_{1}$ (left panel) for neutrons (solid lines) and protons (dashed lines) at three different proton fractions. The momentum is $k=400 \mathrm{MeV}$ and the density and temperature, the same same as in the previous figures.

lines), at the three different proton fractions. The first thing to be observed is that the asymmetry affects mainly the amount of strength exhausted at intermediate energy regions, whereas there is not a noticeable difference at high energies. For $x_{p}=0.5$, i.e., the symmetric case, the quasiparticle energies for neutrons and protons at $k=400 \mathrm{MeV}$, are the same: $\epsilon_{q p}=36.45 \mathrm{MeV}$. When the proton fraction decreases, the quasiparticle energies split. Neutrons become more repulsive $\left(\epsilon_{q p}^{n}=41.88 \mathrm{MeV}\right)$, in contrast with protons $\left(\epsilon_{q p}^{p}=\right.$ $30.86 \mathrm{MeV})$. This behavior is confirmed at $x_{p}=0.04$, resulting in $\epsilon_{q p}^{n}=49.35 \mathrm{MeV}$ and $\epsilon_{q p}^{p}=25.22 \mathrm{MeV}$. In the case of $m_{0}$, since the quasiparticle peak of protons is located at lower energies, the amount of strength exhausted at lower energies is larger for protons than for neutrons. This trend changes at intermediate energies and both strengths merge together for large energies, which is an indication of the fact that, for these momenta and asymmetries, the quasiparticle contributions to the sum rule of neutrons are larger than those of protons, thus explaining why the increase in $m_{0}$ at the quasiparticle pole is larger in the former case. The same type of analysis is valid for $m_{1}$. Notice that, as previously mentioned, the final value of $m_{1}$ is not much affected by the asymmetry and the behavior at high energies is the same for all the cases. Actually, this means that the isospin dependence of the $N N$ correlations is not observed in the result for the sum rule $m_{1}$. However, this isospin dependence can be observed in the convergence of the sum rules at intermediate energies. At those energies, one finds a wider distribution of the single-particle strength for the protons, which reflects the correlations caused by the proton-neutron interaction.

To summarize, we have analyzed the behavior of the energyweighted sum rules of single-particle spectral functions of hot asymmetric nuclear matter. The sum rules are very well fulfilled, because the $T$-matrix approximation itself respects the analytical properties of both the self-energy and the Green's function, in which the sum rules are based. Nevertheless, they are a good test of the numerical consistency of the calculation, which may be helpful, e.g., in deciding the best distribution of the energy mesh points when one has to work with spectral functions. Furthermore, the sum rules are useful to study the isospin dependence of the $N N$ correlations.

Employing realistic $N N$ interactions, an important source of correlations come from the strong components in the neutronproton interaction. As a consequence, one observes in neutronrich matter a larger depletion of the occupation probabilities for protons with momenta below the Fermi momentum than for neutrons. One also finds that these correlations, at this asymmetry, lead to much more attractive quasiparticle energies for protons than those obtained in the HF approximation. The same effect is observed in neutrons, although it is considerably weaker. This shift of the quasiparticle energies tends to lead to more attractive energy-weighted sum rules $m_{1}$. This effect is compensated by the fact that the very same correlations are also responsible for a shift of single-particle strength to high positive energies. As a consequence, the energy integrated sum rules and the isospin splitting in asymmetric nuclear matter for the correlated system yield results that are very close to the $\mathrm{HF}$ ones. This relocation of single-particle strength, however, can be nicely observed in the convergence of the energy-weighted sum rule, which is therefore an indicator of correlation effects.

\section{ACKNOWLEDGMENTS}

The authors are grateful to Angels Ramos for enlightening and fruitful discussions. We would like to acknowledge financial support from the Europäische Graduiertenkolleg Tübingen-Basel (DFG-SNF). This work is partly supported by DGICYT contract BFM2002-01868 and the Generalitat de Catalunya contract SGR2001-64. One of the authors (A. R.) acknowledges the support of DURSI and the European Social Funds.
[1] H. Müther and A. Polls, Prog. Part. Nucl. Phys. 45, 243 (2000).

[2] W. H. Dickhoff and C. Barbieri, Prog. Part. Nucl. Phys. 52, 377 (2004).

[3] M. F. Batenburg, Ph.D. thesis, University of Utrecht, 2001.

[4] D. Rohe et al., Phys. Rev. Lett. 93, 182501 (2004).

[5] M. Baldo, Nuclear Methods and the Nuclear Equation of State, Int. Rev. of Nucl. Phys. (World Scientific, Singapore, 1999), Vol. 9.
[6] S. Fantoni and A. Fabrocini in Microscopic Quantum Many-Body Theories and Their Applications, edited J. Navarro and A. Polls (Springer-Verlag, New York, 1998).

[7] L. P. Kadanoff and G. Baym, Quantum Statistical Mechanics (Benjamin, New York, 1962).

[8] W. D. Kraeft, D. Kremp, W. Ebeling, and G. Röpke, Quantum Statistics of Charged Particle Systems (Akademie-Verlag, Berlin, 1986). 
[9] Y. Dewulf, W. H. Dickhoff, D. Van Neck, E. R. Stoddard, and M. Waroquier, Phys. Rev. Lett. 90, 152501 (2003).

[10] P. Bożek, Phys. Rev. C 59, 2619 (1999).

[11] P. Bożek, Phys. Rev. C 65, 054306 (2002).

[12] T. Frick and H. Müther, Phys. Rev. C 68, 034310 (2003).

[13] T. Frick, H. Müther, A. Rios, A. Polls, and A. Ramos, Phys. Rev. C 71, 014313 (2005).

[14] A. Polls, A. Ramos, J. Ventura, S. Amari, and W. H. Dickhoff, Phys. Rev. C 49, 3050 (1994).
[15] T. Frick, H. Müther, and A. Polls, Phys. Rev. C 69, 054305 (2004).

[16] R. Machleidt, F. Sammarruca, and Y. Song, Phys. Rev. C 53, R1483 (1996).

[17] B. E. Vonderfecht, W. H. Dickhoff, A. Polls, and A. Ramos, Nucl. Phys. A555, 1 (1993).

[18] T. Alm, G. Röpke, A. Schnell, N. H. Kwong, and H. S. Köhler, Phys. Rev. C 53, 2181 (1996).

[19] P. Bożek, Nucl. Phys. A657, 187 (1999). 